\title{
Antifibrinolytic Drugs (Aminocaproic Acid and Tranexamic Acid): Treatment Perspectives for Dental Surgery
}

\author{
Ronald S. Brown ${ }^{1,2,3}$
}

Published online: 1 July 2015

(C) Springer International Publishing AG 2015

\begin{abstract}
The purpose of the review was to evaluate the risks and benefits of antifibrinolytic drugs, aminocaproic acid and tranexamic acid, with respect to dental surgery. The literature supports the utilization of antifibrinolytic drugs particularly with regard to hemophilia and other bleeding dyscrasias. Systemic therapy has potential risks, although blood studies allow for the safe utilization of antifibrinolytics. The use of topical antifibrinolytic drugs demonstrates benefit without significant risk.
\end{abstract}

Keywords Antifibrinolytic - Aminocaproic acid ·

Tranexamic acid $\cdot$ Disseminating intravascular coagulation . Hemophilia $\cdot$ Bleeding dyscrasia

Aminocaproic acid (ACA) and tranexamic acid (TXA) are drugs utilized for enhancing hemostasis, particularly when fibrinolysis contributes to hemorrhaging. Fibrinolytic bleeding may be associated with surgical complications and hematologic disorders such as thrombocytopenia, hemophilia, hepatic cirrhosis, and neoplastic disease [1-3]. Okamoto in 1957 was the first to describe tranexamic acid and aminocaproic acid, synthetic lysine analogues. These drugs are presently

This article is part of the Topical Collection on Oral Medicine

Ronald S. Brown

rbrown@howard.edu; brownrs@mail.nih.gov

1 Department of Comprehensive Dentistry, Howard University College of Dentistry, 600 W Street, NW, Rm. 406, Washington, DC 20059, USA

2 Department of Otolaryngology, Georgetown University Medical Center, Washington, DC, USA

3 Hematology Branch, NHLBI/NIH, Bethesda, MD, USA the most widely used antifibrinolytic drugs. Both act by reversibly blocking the lysine binding sites of plasminogen, thus preventing its activation to plasmin, and therefore stopping the lysis of polymerized fibrin [4].

Oral and maxillofacial surgeons and periodontists have found ACA and TXA to be useful hemostasis adjuncts in oral and periodontal surgery patients with hemophilia and other bleeding dyscrasias. Various case reports and studies have noted relative success with regard to hemophilia, alcoholic cirrhosis of the liver, hereditary angioedema, Glanzmann's thrombasthenia, Bernard-Soulier syndrome, and Trousseau syndrome [5-10].

The terms aminocaproic acid and dentistry (and also tranexamic acid and dentistry) were merged in a PubMed National Library of Medicine search. Ten relevant articles were retrieved, of which there were three studies and seven case reports, one case report reporting two patients. Two of the three studies reported upon hemophilia and one upon the topical application of aminocaproic acid with regard to wound healing.

Both Correa et al. [6] and Sindet-Peterson and Stenbjerg [10] evaluated aminocaproic acid with regard to tooth extraction and other dental surgical therapies in hemophilia patients. Sindet-Peterson and Stenbjerg [10] evaluated three groups of patients (56 subjects) all with congenital deficiencies in factor VIII and factor IX. Patients in the first group received high doses of factor concentrate and systemic TXA. In the second group, the patients received the same therapy as the first group with the addition of topical mouth-rinse TXA. The third group received factor replacement therapy to increase levels to approximately $10 \%$ of the normal value perioperatively, combined with systemic and local (mouth-rinse) TXA. They reported that local antifibrinolytic therapy as a supplement to systemic therapy significantly reduced the incidence of postoperative bleeding. Furthermore, the study results suggested 
that replacement therapy of deficient clotting factors may be reduced during oral surgery in hemophilia patients with utilization of fibrinolysis inhibitors. Correa et al. [6] evaluated 31 hemophilia patients undergoing tooth extraction procedures using fibrin glue and systemic ACA $(200 \mathrm{mg} / \mathrm{kg}$ every $8 \mathrm{~h})$. They observed postsurgical bleeding in six of these patients (one severe, three moderate, and two mild). They evaluated the oral health of their subjects and determined that the status of the subject's oral health did not significantly influence the incidence of postsurgical bleeding.

\section{Pathophysiology: Coagulation and Fibrin}

The third stage of hemostasis is the coagulation phase which is dependent upon the generation of thrombin and fibrin. Multiple proteins synthesized by the liver are necessary which include fibrinogen, prothrombin, and factors V, VII, IX, X, XI, XII, and XIII. There are three separate pathways involved (1) intrinsic, (2) extrinsic, and (3) an activating common pathway supported by both the intrinsic and extrinsic pathways [11, 12] (see Fig. 1).

\section{Intrinsic Pathway}

Factor XII initiates the intrinsic pathway and involves an interaction between factor XII and factor XI as factor XII is converted to factor XIIa. Factor XIIa aids in the activation of factor XI to factor XIa. Factor XIa aids in

\section{Coagulation Cascade}

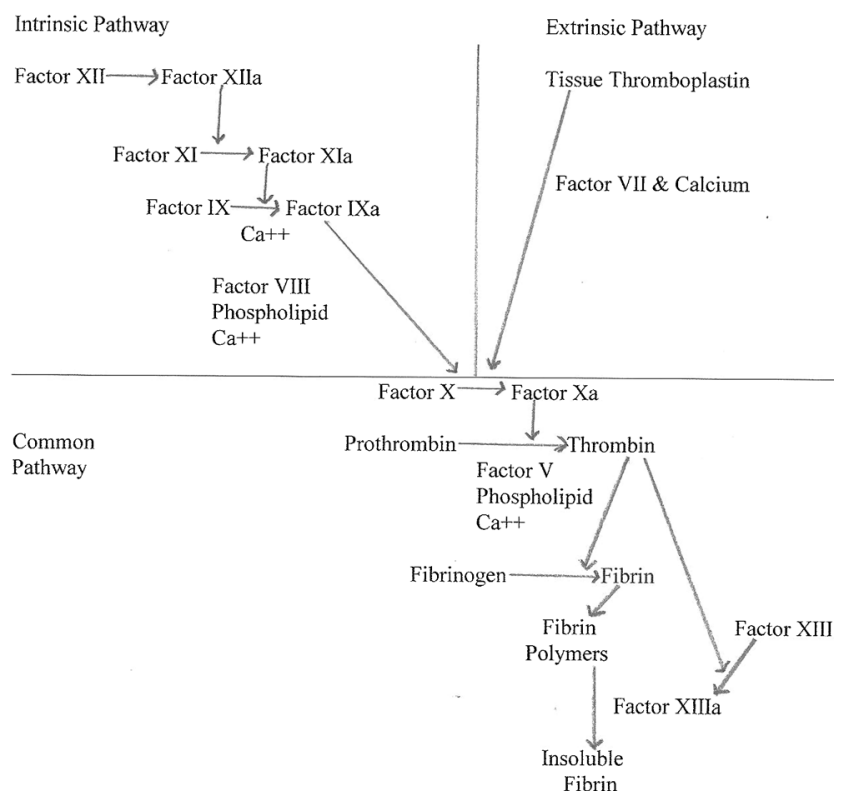

Fig. 1 Coagulation cascade the activation of factor IX to factor IXa. A complex is then formed between factor IXa and factor VIII. This reaction requires $\mathrm{Ca}++$ and phospholipid.

\section{Extrinsic Pathway}

The extrinsic pathway involves factor VII and calcium acting upon tissue thromboplastin which then in combination of the end products of the intrinsic pathway (factor IXa, factor VIII, phospholipid, and calcium) activates factor $\mathrm{X}$ to factor $\mathrm{Xa}$.

\section{The Common Pathway}

Factor Xa (with factor V, phospholipid, and calcium) then allows the conversion of prothrombin to thrombin. Thrombin then allows the conversion of fibrinogen to fibrin and fibrin becomes fibrin polymers which with factor XIIIa allows the conversion of fibrin polymers to insoluble fibrin. Thrombin also aids in the conversion of factor XIII to factor XIIIa which is necessary in the conversion of fibrin polymers to insoluble fibrin [11, $12]$.

\section{The Fibrinolytic System}

The beginning of fibrinolytic system involves the activation of factor XII to factor XIIa (see Fig. 2). Factor XIIa along with high molecular weight kininogen is necessary for the conversion of prekallikrein to kallikrein. Kallikrein (along with plasminogen activator) is necessary for the conversion of plasminogen to plasmin. Plasmin is necessary for the conversion of fibrin to fibrin degradation products [11-14].

\section{Fibrinolytic System}

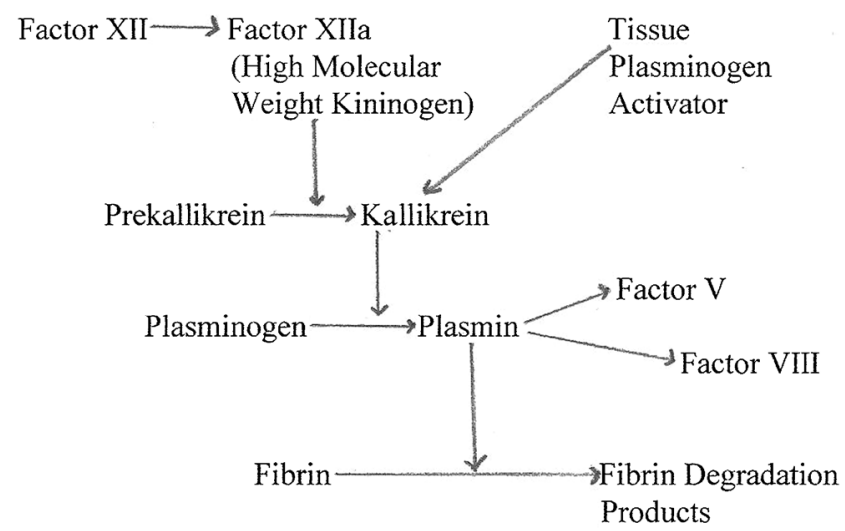

Fig. 2 Fibrinolytic system 


\section{Fibrinolytic Disorders, Coagulation Factors, and Disseminated Intravascular Coagulation}

Fibrinolysis may result in hemorrhage due to the enhancement of clot breakdown. A deficiency in plasminogen activator inhibitor has the potential to result in primary fibrinolysis. Factor XIII stabilizes fibrin clots and a deficiency of factor XIII may lead to fibrinolysis and bleeding. Hematologic disorders impairing the fibrinolytic system can also lead to excessive clotting and thrombosis due to retarded clot breakdown. The treatment for primary fibrinolysis is fresh frozen plasma (FFP) and antifibrinolytics such as ACA and TXA. A number of fibrinolytic conditions may present bleeding issues related to complications in oral and periodontal surgery. Of course, other conditions and drugs which may cause primarily coagulation disorders, such as immune thrombocytopenic purpura, aplastic anemia, myelodysplastic disorder, nonsteroidal anti-inflammatory drugs, warfarin sodium, and dabigatran, may present hemostatic control issues for periodontal and oral surgery clinicians due to decreased availability or function of platelets or the decreased ability of vitamin $\mathrm{K}$ to function or the inhibition of thrombin. Primary fibrinolysis is a normal bodily process which prevents blood clots that occur naturally from increasing in size and causing blockage. Secondary fibrinolysis refers to the breakdown of clots due to medicine, a medical disorder, or other causes such as bacterial infections, cancer, tissue hypoxia, and intense exercise. Related problematic bleeding disorders and associated deficient factors include hemophilia A (factor VIII), hemophilia B (factor IX), von Willebrand's disease (defect within factor VIII), liver disease (decreased vitamin Kdependent factors II, VII, IX, and X), and Glanzmann's thrombasthenia (congenital platelet disorder). The Trousseau syndrome is a form of disseminated coagulation, venous thrombosis, and hypercoagulability, associated with cancer. The pathological phenomenon of clots forming, resolving, and then appearing again elsewhere in the body has also been named thrombophlebitis migrans or migratory thrombophlebitis, as opposed to plain thrombophlebitis in one location $[5,11-16]$.

Acute disseminated intravascular coagulation (DIC) is a very serious condition with a high risk of death. This condition is characterized by systemic intravascular activation of coagulation and the deposition of fibrin. Major activation of coagulation may deplete platelets and coagulation factors, leading to uncontrollable hemorrhage and lethal consequences. Furthermore, it is important for the clinician to properly diagnose secondary fibrinolysis associated with DIC, as this condition could lead to thrombosis, and thrombotic complications as fibrin deposition has the potential to contribute to multiple-organ failure [1, 17].

\section{Determination of Bleeding Complications}

Tests associated with the evaluation of bleeding complications for dental patients undergoing surgery include (1) complete blood count $(\mathrm{CBC})$, which includes the number of platelets, and platelets are necessary for coagulation and too many platelets can be adverse for too much clotting; (2) blood smear which can determine damaged blood cells; (3) bleeding time (BT), a test to assess platelet function, and determine the time to stop bleeding; (4) prothrombin time (PT) which is a measure of the extrinsic pathway and for determining the amount of time for clotting; (5) international normalization ratio (INR) which is also a measure of the extrinsic pathway and for determining the amount of time for clotting; (6) partial prothrombin time (PTT) which is a monitor of both the intrinsic and common pathways and for determining the amount of time for clotting; (7) serum fibrinogen which measures the level of fibrinogen in the serum; (8) fibrin degradation products, a measurement of degradation products in the serum after blood clot dissolution; (9) protamin paracoagulation test, which is positive in DIC and negative in the presence of primary fibrinolysis; and (10) euglobulin clot lysis test, which is abnormal in primary fibrinolysis, but normal in DIC. ACA and TXA should not be used in the presence of DIC without also using heparin due to the possibility of causing thromboemboli [5, 11-16].

Yepes et al. [5], in addressing treatment planning issues for patients with DIC and hypercoagulability syndromes, has suggested the following: (1) hematology consultation, (2) consideration of the risk benefit analysis regarding the proposed dental surgical procedures, (3) consideration for anticoagulation attenuation, (4) consideration for the utilization of local measures for control of hemorrhage, (5) an understanding regarding the potential of postoperative medications to interfere with anticoagulation medications, (6) an understanding of potential problems with utilization of the Trendelenburg or subsupine positions, and (7) consideration for hospitalization.

Wardrop et al. [1] reviewed the literature with regard to the utilization of antifibrinolytics for the prevention of bleeding in patients with hematologic disorders. They reported that the evidence available for the use of antifibrinolytics in hematology patients is very limited. They reported that the only available data suggested that TXA and ACA may be useful adjuncts to platelet transfusions so that platelet use, and the complications associated with their use, can be reduced. However, the study populations were too small to assess whether antifibrinolytics increased the risk of thromboembolic events. Their conclusion was that large, high-quality RCTs are required before antifibrinolytics can be demonstrated to be efficacious and safe in widespread clinical practice.

While the utilization of ACA and TXA is supported within the literature for the control of hemorrhage in dental patients 
with a number of bleeding dyscrasias (hemophilia A, hemophilia B, von Willebrand's disease (defect within factor VIII), liver disease (decreased vitamin K-dependent factors II, VII, IX, and X), and Glanzmann's thrombasthenia), ACA and TXA therapy should not be utilized when there is evidence of an active intravascular clotting process. These drugs should not be utilized without a definitive diagnosis typically determined by laboratory findings indicating hyperfibrinolysis. It is important to distinguish whether or not the cause of the bleeding is due to primary fibrinolysis or DIC, as hemorrhage secondary to DIC should not be treated with any antifibrinolytic agent (ACA or TXA) unless there is both a bleeding tendency and a systemic fibrinogenolysis utilization. ACA and TXA may increase the risk of DIC. Appropriate blood studies include platelet count, protamin paracoagulation test, and the euglobulin clot lysis test. Platelets are decreased in DIC but normal in primary fibrinolysis. The protamine paracoagulation test is positive in DIC and negative for primary fibrinolysis. The euglobulin clot lysis test is abnormal in primary fibrinolysis and normal in DIC [17-19] (see Table 1).

Side effects and overdose of antifibrinolytics are associated with embolytic events. Such adverse reactions include sudden numbness or weakness, particularly of one side of the body; sudden headache; confusion; seizures; problems with vision, speech, and/or balance; pain or swelling in one or both legs; and muscle pain, tenderness, or weakness [20-22].

Sindet-Pedersen and Stenbjerg [10] investigated the utilization of local antifibrinolytic treatment with TXA on hemophiliac dental patients. They reported that local TXA therapy significantly reduced the incidence of postoperative bleeding without increasing significant risks. Padovan et al. [7] evaluated wound healing repair after topical ACA application to extraction sites in a warfarin drug animal (rats) study. They reported that through histological analysis, it was possible to

Table 1 Correlations

\begin{tabular}{lllll}
\hline & PTT & PT/INR & BT & PC \\
\hline Factor VII def & WNL & + & WNL & WNL \\
Factor VIII def & + & WNL & WNL & WNL \\
Factor IX def & + & WNL & WNL & WNL \\
Factor XI def & + & WNL & WNL & WNL \\
LD & WNL & + & WNL & WNL \\
WSAC & WNL & + & WNL & WNL \\
SLD & + & + & + & - \\
VWD & + or WNL & WNL & + & - or WNL \\
DIC & + & + & + & -
\end{tabular}

Def deficiency, $L D$ liver disease, WSAC warfarin sodium anticoagulation, $S L D$ severe liver disease, $V W D$ von Willebrand's disease, $D I C$ disseminated intervascular coagulation, $P T T$ partial thromboplastin time, $P T / I N R$ prothrombin time/international normalization rate, $B T$ bleeding time, $P C$ platelet count, $W N L$ within normal limits, + increased, - decreased observe that tooth socket irrigation with ACA advanced wound healing repair.

In conclusion, antifibrinolytic drugs aminocaproic acid and tranexamic acid may be useful adjunctive pharmacotherapeutics in the treatment of dental patients with a number of bleeding dyscrasias. It is important to utilize proper diagnostic and therapeutic diligence with regard to the systemic administration of aminocaproic acid and tranexamic acid as these systemic pharmacotherapeutics have potential negative consequences.

\section{Compliance with Ethics Guidelines}

Conflict of Interest Ronald S. Brown declares that he has no conflict of interest.

Human and Animal Rights and Informed Consent This article does not contain any studies with human or animal subjects performed by the author.

\section{References}

1. Wardrop D et al. Antifrinolytics (lysin analogues) for the prevention of bleeding in patients with haematological disorders (review). Cochrane Database Syst Rev. 2013;7:CD009733.

2. Van Sickels NJ, Hunsaker RB, Van Sickels JE. Hereditary angioedema: treatment management, and precautions in patients presenting for dental care. Oral Surg Oral Med Oral Pathol Oral Radiol Endod. 2010;109(2):168-72.

3. Hartman MJ, Caccamese Jr JF, Bergman SA. Perioperative management of a patient with Bernard-Soulier syndrome for third molar surgery. Oral Surg Oral Med Oral Pathol Oral Radiol Endod. 2007;103(5):626-9.

4. Okamoto $\mathrm{S}$ et al. Enzyme controlling medicines: introduction. Semin Thromb Hemost. 1997;23(6):493-501.

5. Yepes JF et al. Hypercoagulability syndromes: what the dentist needs to know. Oral Surg Oral Med Oral Pathol Oral Radiol Endod. 2007;104(1):3-11.

6. Correa MEP et al. Clinical impact of oral health indexes in dental extraction of hemophilic patients. J Oral Maxillofac Surg. 2006;64(5):785-8.

7. Padovan LEM et al. Fibrin adhesive implant in wound healing repair of dental sockets with topical application of epsilon aminocaproic acid: histological analysis. J Biomed Mater Res B Appl Biomater. 2005;73B(2):209-13.

8. Gomes MF et al. Surgical and clinical management of a patient with Glanzmann thrombasthenia: a case report. Quintessence Int. 2004;35(8):617-20.

9. Bisch FC et al. Dental considerations for a Glanzmann's thrombasthenia patient: case report. J Periodontol. 1996;67(5): 536-40.

10. Sindet-Pedersen S, Stenbjerg S. Effect of local antifibrinolytic treatment with tranexamic acid in hemophiliacs undergoing oral surgery. J Maxillofac Surg. 1986;44(9):703-7.

11. Patton LL. Bleeding and clotting disorders. In: Greenberg MS, Glick M, editors. Burket's oral medicine: diagnosis and treatment. 10th ed. Hamilton: B.C. Decker; 2003. p. 411-23.

12. Sindet-Pedersen S. Haemostasis in oral surgery - the possible pathogenetic implications of oral fibrinolysis on bleeding. Dan Med Bull. 1991;38(6):427-43. 
13. Levi $\mathrm{M}$ et al. Infection and inflammation and the coagulation system. Cardiovasc Res. 2003;60(1):26-39.

14. Levi $\mathrm{M}$ et al. Advances in the understanding of the pathophysiologic pathways of disseminated intravascular coagulation result in more insight in the clinical picture and better management strategies. Semin Thromb Hemost. 2001;27(6):569-75.

15. Rickles FR, Edwards RL. Activation of blood coagulation in cancer: trousseau's syndrome revisited. Blood. 1983;62(1):12-32.

16. Schafer AI, Levine MN, Konkle BA, Kearon C. Thrombotic disorders: diagnosis and treatment. Thrombotic disorders: diagnosis and treatment. Hematol AM Soc Hematol Educ Program. 2003;520-39.

17. Levi M, de Jonge E, ten Cate H. Disseminated intravascular coagulation. Ned Tijdschr Geneeskd. 2000;144(10):470-5.
18. Sistino JJ, Richards C. The additive effects of antifibrinolytics: dangers in the OR. J Extra Corpor Technol. 1999;31(2):97-101.

19. Okajima $\mathrm{K}$ et al. Direct evidence for systemic fibrinogenolysis in patients with acquired $\forall 2$-plasmin inhibitor deficiency. Am J Hematol. 1994;45(1):16-24.

20. Bell D et al. Tranexamic acid in cardiac surgery and postoperative seizures: a case report series. Heart Surg Forum. 2010;13(4):E2579.

21. Blanco-Carrion $\mathrm{J}$ et al. Morbidity and economic complications following mucogingival surgery in a hemophiliac HIV-infected patient: a case report. J Periodontol. 2004;75(10):1413-6.

22. Dunn CJ, Goa KL. Tranexamic acid: a review of its use in surgery and other indications. Drugs. 1999;57(6):1005-32. 